\title{
Cognitive effects of pituitary tumours and their treatments: two case studies and an investigation of 90 patients
}

\author{
Elizabeth M Guinan, Clara Lowy, Nicola Stanhope, Philip D R Lewis,
} Michael D Kopelman

\begin{abstract}
Objectives-Two case studies are reported of patients with pituitary adenomas who had been treated with trans-sphenoidal surgery, one with and one without adjunctive radiotherapy, in whom memory impairment was found. Further to this, neuropsychological investigations of 90 patients were carried out (1) to establish the prevalence of such deficits, and (2) to try to determine their cause.
\end{abstract}

Methods-Two case studies are described. For the expanded study, patients were recruited from the data base of the endocrinology department of St Thomas's Hospital, London, if they had previously been treated for a pituitary adenoma in the past 30 years. Ninety patients were contacted and assessed with a wide range of neuropsychological tests. They were divided into five treatment groups: those who had received transfrontal surgery with radiotherapy, trans-sphenoidal surgery with or without radiotherapy, radiotherapy only, and a bromocriptine therapy group, as well as a group of 19 healthy control subjects matched for age and sex. Results-In the two patients presented, both showed severe memory impairments compared with their intact intellectual ability. The more severely affected patient had received adjunctive radiotherapy, and superimposition of the $90 \%$ isodose fields on a postoperative MRI examination suggested involvement of the diencephalic structures. In the group study, significant deficits in anterograde memory were also obtained on two measures (WMS-R, RMT) for all patient groups when compared with the healthy controls, although these impairments varied in degree and were less in the bromocriptine group. However, the individual surgical and radiotherapy treatment groups did not differ significantly from one another. By contrast, general intellectual function (IQ) remained intact for all groups, as did performance on supplementary cognitive tests, including measures of frontal lobe or "executive" function, language comprehension, and speed of mental processing. Psychiatric morbidity and tumour aetiology did not seem to relate to the presence of memory deficits.

Conclusions-Anterograde memory deficits were seen in the two case studies and in all our treatment groups when compared with the healthy controls, and these occurred in the context of preserved intellectual function. The present findings suggest that these memory deficits result from treatment rather than from the underlying tumour, but there was no difference between the effects of surgery and radiotherapy. It is suggested that they result from damage to diencephalic structures implicated in memory.

(F Neurol Neurosurg Psychiatry 1998;65:870-876)

Keywords: pituitary tumours; memory impairments; radiotherapy; surgery

Pituitary adenomas account for about $10 \%$ of intracranial tumours and, although nearly always benign in nature, usually require treatment to alleviate pressure effects and hormone hypersecretion. Many structures adjacent to the pituitary are known to be important for normal memory processing and are, therefore, at risk of incidental damage from the adenoma itself or from the surgical or radiotherapy procedures employed in treatment.

There are reports of cognitive deficits in patients treated with radiotherapy for a nasopharyngeal carcinoma, ${ }^{1-3}$ but only recently have a few detailed neuropsychological studies been reported for pituitary tumours. Grattan-Smith et $a l^{4}$ performed neuropsychological testing in a group of patients with pituitary adenoma and reported memory impairments in these patients when compared with a control group. However, their study concentrated on the putative effect of radiotherapy on cognitive function, and they did not give a detailed breakdown of cognitive outcome for different treatment strategies. There are, however, many possible contributory factors that may be responsible for cognitive impairments in patients who have been diagnosed and treated for a pituitary tumour. For example, there may be a mass effect from the original tumour on the third ventricle and diencephalic structures which are in close proximity to the pituitary region. Luria ${ }^{5}$ described an amnesic syndrome in patients with large tumours invading the diencephalon, and Lishman ${ }^{6}$ reviewed claims that cognitive deficits are seen in patients with pituitary tumours which have large suprasellar extensions. Hormone hypersecretion by certain types of pituitary adenoma may also have cognitive or psychiatric effects as reported, for example, in Cushing's disease. ${ }^{78}$ 
In the present paper, we report two case histories of patients with severe anterograde memory impairment, one of whom had been treated with trans-sphenoidal surgery alone and the other with surgery and adjunctive radiotherapy. We then describe the findings from a neuropsychological investigation of 90 patients, which was carried out in response to these two cases. We wished to establish the frequency and severity of cognitive deficits in these patients and also to differentiate the specific effects of different surgical routes (transsphenoidal or transfrontal), radiotherapy, and bromocriptine therapy on cognitive function, after controlling for type of tumour and time since treatment.

\section{Case histories}

PATIENT 1

A 49 year deputy headmistress was diagnosed as having a chromophobe adenoma after progressive bitemporal visual field loss particularly affecting the right eye. There was a large suprasellar extension, the tumour being measured as $24 \times 21 \times 16 \mathrm{~mm}$ on a preoperative MRI, and there was displacement of the optic chiasm, deformation of the third ventricle, and some lateral spread on the right side. The patient underwent a trans-sphenoidal hypophysectomy in March 1988 and a course of postoperative radiotherapy in May and June 1988. Pituitary irradiation was performed using an $8 \mathrm{MeV}$ linear accelerator which delivered a total treatment dose of 4680 cGy in 25 daily fractions over five weeks. A three field technique was used whereby treatment was delivered via two lateral ports, one to each temporal region, and an anterior port angled so that the inferior edge of the beam was above the eyes. This allowed the maximum required treatment dose to be delivered to the pituitary region, while reducing the relative dose to the normal tissues of the frontal and temporal lobes.

By 1991, now aged 52 years, our patient was becoming increasingly lethargic and quick to tire and her endocrine profile showed evidence of hypopituitarism and in particular of growth hormone deficiency. Despite correction of her hypopituitarism, including administration of growth hormone, ${ }^{9}$ the patient complained of increasing lethargy, and was referred to our neuropsychiatry clinic in July 1992. At her first psychiatric assessment, she described characteristic features of depression, including depressed mood, irritability, and sleep and appetite disturbances, and she was prescribed an antidepressant (desipramine). At her second assessment, however, the patient emphasised that it was only when working at school that she became depressed, and that she was fine at weekends and on holidays. The patient seemed highly intelligent and articulate but, because of the context dependent nature of her depression, we decided to carry out a cognitive assessment.

PATIENT 2

Our second patient received a trans-sphenoidal hypophysectomy for Cushing's disease secondary to a pituitary adenoma without adjunctive

Table 1 Cognitive test scores

\begin{tabular}{|c|c|c|c|c|}
\hline & \multicolumn{2}{|l|}{ Patient 1} & \multicolumn{2}{|l|}{ Patient 2} \\
\hline \multicolumn{5}{|c|}{ General cognitive function; Wechsler adult intelligence scale-revised (WAIS-R): } \\
\hline Full scale IQ & \multicolumn{2}{|c|}{138} & \multicolumn{2}{|l|}{124} \\
\hline Verbal IQ & \multicolumn{2}{|l|}{132} & \multicolumn{2}{|l|}{132} \\
\hline Performance IQ & \multicolumn{2}{|c|}{136} & \multicolumn{2}{|l|}{109} \\
\hline \multicolumn{5}{|c|}{ Anterograde memory; Wechsler memory scale-revised (WMS-R): } \\
\hline General memory & \multicolumn{2}{|c|}{89} & \multicolumn{2}{|l|}{97} \\
\hline Delayed memory & \multicolumn{2}{|l|}{91} & \multicolumn{2}{|l|}{74} \\
\hline Verbal memory & \multicolumn{2}{|l|}{83} & \multicolumn{2}{|l|}{107} \\
\hline Visual memory & \multicolumn{2}{|l|}{105} & \multicolumn{2}{|l|}{85} \\
\hline Attention & \multicolumn{2}{|c|}{126} & \multicolumn{2}{|l|}{121} \\
\hline \multicolumn{5}{|c|}{ Recognition memory; recognition memory test (RMT): } \\
\hline Words raw score & $40 / 50$ & (10.3 percentile) & $44 / 50$ & (63.8 percentile) \\
\hline Faces raw score & $42 / 50$ & (23.4 percentile) & $37 / 50$ & (7.6 percentile) \\
\hline \multicolumn{5}{|c|}{ Retrograde memory } \\
\hline \multicolumn{5}{|c|}{ Autobiographical memory inventory (AMI): } \\
\hline Facts & & Maximum $=21$ for each period & & \\
\hline Childhood & 17 & Normal & 21 & Normal \\
\hline Early adult life & 21 & Normal & 21 & Normal \\
\hline Recent life & 21 & Normal & 14.5 & Definately abnormal \\
\hline Incidents & & Maximum $=9$ for each period & & \\
\hline Childhood & 9 & Normal & 9 & Normal \\
\hline Early adult life & 6 & Normal & 6 & Borderline impairment \\
\hline Recent life & 9 & Normal & 7 & Normal \\
\hline \multicolumn{5}{|c|}{ Tests of language comprehension and speed of mental processing: } \\
\hline Graded naming test & $27 / 30$ & Normal & $24 / 30$ & Normal \\
\hline \multicolumn{5}{|c|}{ Paced auditory serial addition test } \\
\hline $2.4 \mathrm{~s}$ & $56 / 60$ & $(46(5))^{\star}$ & $33 / 60$ & \\
\hline $2.0 \mathrm{~s}$ & $52 / 60$ & $(40(7))^{\star}$ & $31 / 60$ & \\
\hline $1.6 \mathrm{~s}$ & $36 / 60$ & $(32(8))^{\star}$ & $34 / 60$ & \\
\hline $1.2 \mathrm{~s}$ & $29 / 60$ & $(22(5))^{\star}$ & $25 / 60$ & \\
\hline \multicolumn{5}{|c|}{ Speed and capacity of language processing test: } \\
\hline Sentences in $2 \min (\mathrm{n})$ & $91 / 100$ & (95th percentile) & $84 / 100$ & (95th percentile) \\
\hline Spot the word & $58 / 60$ & (95th percentile) & $56 / 60$ & (90th percentile) \\
\hline Frontal lobe or tests of execu & tion: & & & \\
\hline FAS & 52 & (77-96th percentile) & 75 & (96th percentile) \\
\hline Cognitive estimations & 10 & (Moderate impairment) & 2 & Normal \\
\hline Modified card sorting test & & & & \\
\hline Catagories (n) & 4 & (Moderate impairment) & 5 & (Normal $=5-6$ categories) \\
\hline Errors & 14 & & 9 & \\
\hline Perseverative errors & 2 & & 2 & \\
\hline
\end{tabular}

${ }^{\star}$ Comparison mean scores for healthy subjects, reported in Lezak ${ }^{33}$ and adapted from Gronwall and Wrightson. ${ }^{19}$ 


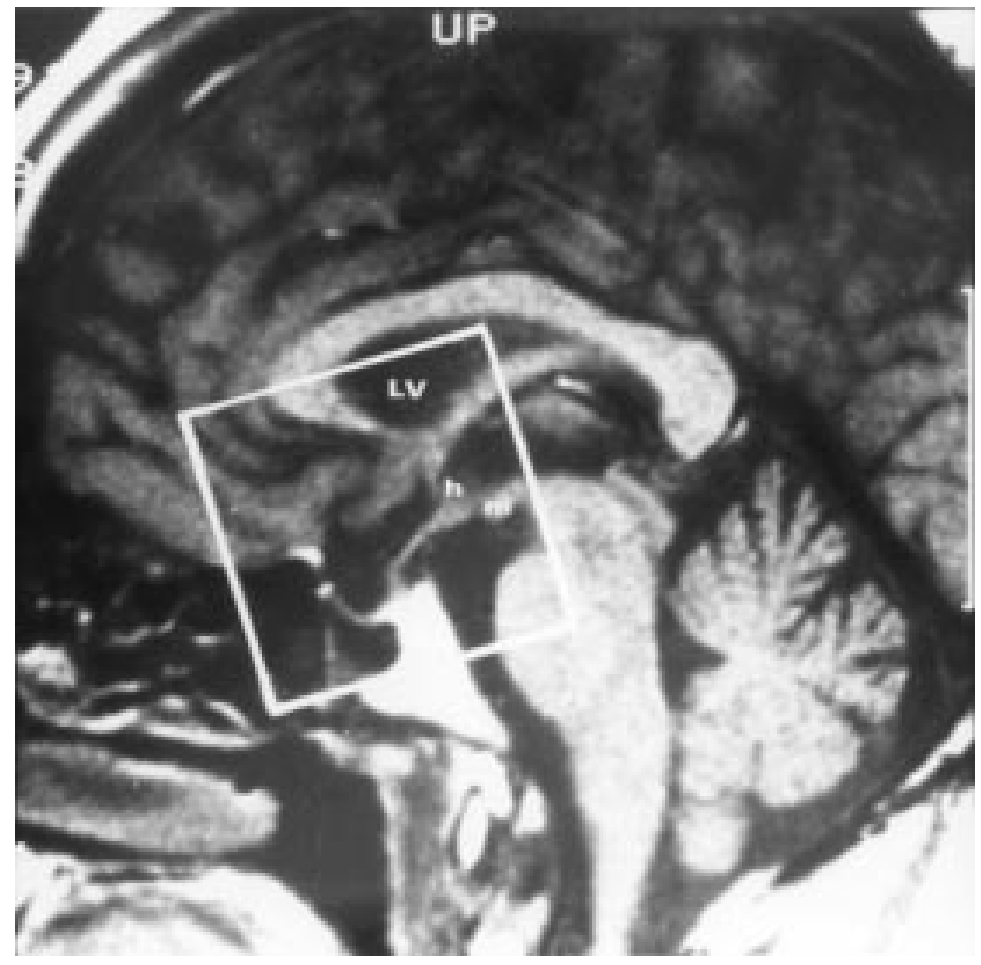

Figure 1 Plot of the fields of maximum irradiation in patient 1. The field markers from the lateral $x$ ray simulation film have been superimposed on the patient's own postoperative sagittal MRI, a cut $3 \mathrm{~mm}$ from the midline in the right hemisphere. The three treatment fields were set at $5 \times 5 \times 5 \mathrm{~cm}$ so that the total tissue volume of the $90 \%$ isodose region was $125 \mathrm{~cm}^{3}$ and this is illustrated. The lines are shown to be square because the treatment port were unshaped (although there may have been slight rounding at the corners). The volume of tissue which received $99 \%$ of the total treatment dose measured about $34 \%$ of the region shown in the figure $\left(42.5 \mathrm{~cm}^{3}\right)$, and that receiving $100 \%$ of the dose was considerably smaller, about $8 \%$ of the region shown $\left(10.0 \mathrm{~cm}^{3}\right)$. Structures within the $90 \%$ tissue volume included the mammillary bodies $(m)$, the hypothalamus $(h)$, the column of the fornix, and the mammillothalamic tract bilaterally, as well as the anterior commissure and the optic tract. $L V=$ lateral ventricle.

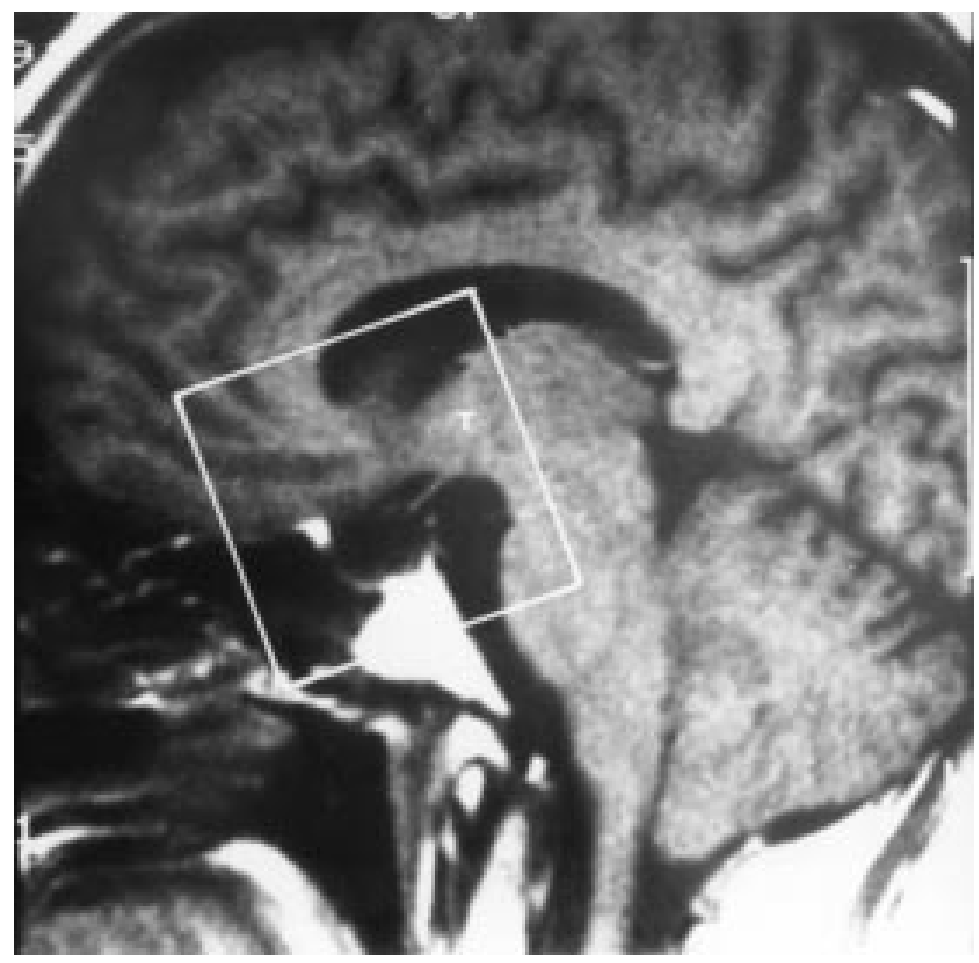

Figure 2 A sagittal slice $1 \mathrm{~cm}$ to the right of the midline shows the $90 \%$ isodose region as before, here indicating involvement of the anterior portion of the thalamus $(T)$. radiotherapy in February 1988. When first seen, she manifested hirsutism, mood change, and cushingoid physical features, and she complained of memory loss. She had made a good postoperative recovery but, because of a radical resection of her microadenoma, she developed panhypopituitarism. She was tested on the same neuropsychological test battery as patient 1 in 1994, when aged 63 years, and she was receiving hormonal replacement therapy at the time of her assessment, including growth hormone. At $458 \mathrm{nmol} / 1$ her cortisol concentrations were slightly raised, and with an insulin-like growth factor of $17.3 \mathrm{nmol} / 1$ her growth hormone concentrations were towards the lower end of the normal range.

NEUROPSYCHOLOGICAL FINDINGS

Table 1 shows the neuropsychological test battery and results for patients 1 and 2. On the WAIS-R, ${ }^{10}$ both patients showed preserved intellectual ability, with current full scale IQ scores of 138 for patient 1("very superior" range) and 124 for patient 2 ("superior" range). By contrast, they both showed deficits in anterograde memory on the Wechsler memory scale- revised (WMS-R), ${ }^{11}$ relative to their current IQ. Patient 1 had a general memory quotient of 89 points, which was more than 3 SD below her full scale IQ score, and the general memory quotient of patient 2 was 97 , a decrement approaching $2 \mathrm{SD}$. On the logical memory and visual reproduction subtests of the WMS-R, patient 1 scored poorly on the logical memory stories (14th percentile) but normally on visual reproduction (78th percentile), whereas patient 2 showed a moderate impairment on immediate visual reproduction (68th percentile) and severe impairment on both tests at a 30 minute delay relative to her IQ (48th percentile for logical memory, and 1st percentile for visual reproduction). On the recognition memory test, ${ }^{12}$ patient 1 was impaired for both words and faces, scoring at the 10th and $23 \mathrm{rd}$ age related percentile respectively, and patient 2 was severely impaired for faces (scoring at the 7 th percentile). Whereas patient 1 showed moderate impairment on the cognitive estimates ${ }^{13}$ and modified card sorting tests, ${ }^{14}$ she showed superior performance on the FAS verbal fluency test, ${ }^{15}{ }^{16}$ and patient 2 showed a normal performance on all of the "executive" function tests. On measures of language ${ }^{17} 18$ and speed of mental processing, ${ }^{19}$ both patients scored within the normal or superior range.

\section{RADIOLOGICAL FINDINGS}

Patient 1 underwent whole brain MRI and fluorodeoxy glucose PET (as part of a parallel research study), but on visual inspection the scans did not disclose any abnormality beyond the pituitary. Brain MRI for patient 2 also did not show any pathology outside the pituitary region.

Figures 1 and 2 plot the fields of maximum irradiation in patient 1 . The field markers from the lateral $x$ ray simulation film have been superimposed on the patient's own postoperative sagittal MRI. The three treatment fields, 
delineated by the $90 \%$ isodose curve, were set at $5 \times 5 \times 5 \mathrm{~cm}$ so that the total tissue volume of the $90 \%$ isodose region was $125 \mathrm{~cm}^{3}$ and this is illustrated in the figures. The lines are shown to be square because the treatment ports were unshaped (although there may have been slight rounding at the corners). The volume of tissue which received $99 \%$ of the total treatment dose measured about $34 \%$ of the region shown in the figures $\left(42.5 \mathrm{~cm}^{3}\right)$, and that receiving $100 \%$ of the dose was considerably smaller, about $8 \%$ of the region shown $\left(10.0 \mathrm{~cm}^{3}\right)$. As figs 1 and 2 illustrate, structures within the $90 \%$ tissue volume included the mammillary bodies, the hypothalamus, the columns of the fornix, the mammillothalamic tract, and the anterior portion of the thalamus bilaterally, as well as the anterior commissure and the optic tract.

\section{Discussion}

These two patients, who had been diagnosed and treated for pituitary tumours, showed moderate to severe memory impairment, sufficient to cause distress and problems in everyday life. However, the putative cause of these memory deficits was difficult to tease apart. Patient 1, who showed the more severe impairment, had a large pituitary tumour which initially exerted pressure effects on the optic chiasm. She also received postoperative radiotherapy to brain structures known to be critical for memory formation, and there are reports in the literature of cognitive impairments in patients who have received cranial radiotherapy for other types of tumour. ${ }^{2}{ }^{20} \mathrm{On}$ the other hand, patient 2 displayed fairly severe memory impairments in the absence of either obvious pressure effects or irradiation. The clinical literature pays little attention to the long term effects on cognitive function of either transfrontal or trans-sphenoidal surgery. This may be because postoperative complications of surgery are usually immediately and readily apparent. Some effects, however, may be relatively subtle and easily missed, or may be delayed in their appearance, and consequently they may not get reported in the clinical literature. Both of our patients received transsphenoidal excision of their tumours, which is less invasive than the transfrontal route. Memory impairment after surgery without radiotherapy may result from damage to the same structures as are implicated within the $90 \%$ dose of irradiation, although the mechanism of this remains unclear. To examine these issues further, we carried out a retrospective study of 109 subjects, including 90 patients.

\section{Retrospective study of 90 patients \\ SUBJECTS}

Patients were recruited from the database of the endocrine department of St Thomas's Hospital, if they had been treated for a pituitary adenoma of any aetiology between 1966 and 1993, and were between 18 and 70 years of age. The final sample of 90 patients represented about half of the patients with a pituitary tumour who were eligible for the study. Patients were excluded from the study if (1) they had residual visual field defects of a severity that could have compromised their performance on the cognitive tests, (2) they had received any treatment procedure on more than one occasion, (3) they had any concurrent pathology such as cerebrovascular or metastatic disease, or (4) they required continuing anticonvulsant therapy. A further requirement of patient selection from the database was that the treatment groups were matched as closely as possible for mean age and sex distribution.

Patients included in the study were distributed in the following groups: those who had received transfrontal hypophysectomy and postoperative radiotherapy (20 patients), transsphenoidal hypophysectomy and postoperative radiotherapy (21 patients), trans-sphenoidal hypophysectomy without radiotherapy (21 patients), radiotherapy treatment only (10 patients), and bromocriptine drug therapy (18 patients). We were unable to find enough patients who had received transfrontal hypophysectomy in the absence of postoperative radiotherapy for a viable group, and patients who had received radiotherapy in the absence of surgery were also more difficult to recruit. A group of 19 healthy controls were also included in the study who, wherever possible, were selected from the friends and family of the patients to match for age and socioeconomic factors, such as education and occupation.

\section{PROCEDURE}

Important clinical details were recorded from the patients' case notes, and included type and size of tumour, pretreatment and posttreatment hormone concentrations, any postoperative complications, the various radiotherapy treatment indices (dose and fields), time since treatment, and current medication. Each patient was assessed on the same neuropsychological test battery as used in the two patients above (table 1), which included a reading test to assess estimated premorbid IQ (NART-R), ${ }^{21}$ a measure of retrograde memory (AMI), ${ }^{22}$ and some psychiatric scales to assess anxiety, depression, and personality traits. ${ }^{23-27}$ The test presentation order was counterbalanced to avoid any confounding effects of test anxiety and fatigue experienced by the subject during the testing sessions. Because the case studies had suggested deficits in anterograde memory (WMS-R, RMT) relative to IQ (WAIS-R), these were regarded as our main outcome measures, and the other tasks were supplementary measures to determine the breadth and correlates of impaired performance.

\section{Results}

The results for the main outcome measures are shown in table 2 . There was no significant difference between the groups in terms of their estimated premorbid IQ, as determined by a reading test, ${ }^{21}$ although there was a trend for the radiotherapy group to score lowest ( $F$ $(5,103)=2.17 \mathrm{p}<0.065)$. There was a significant difference $(F(5,103)=4.02 \mathrm{p}<0.002)$ between the groups in terms of their current full scale IQ (WAIS-R). The mean scores of the 
Table 2 Mean scores for main outcome measures

\begin{tabular}{|c|c|c|c|c|c|}
\hline Treatment group & Age & $\begin{array}{l}\text { Estimated premorbid } \\
I Q(N A R T-R)\end{array}$ & $\begin{array}{l}\text { Current IQ } \\
\text { (WAIS-R) }\end{array}$ & $\begin{array}{l}\text { General memory } \\
(W M S-R)\end{array}$ & $\begin{array}{l}\text { Delayed memory } \\
(W M S-R)\end{array}$ \\
\hline $\mathrm{TF}+\mathrm{RT}$ & 50.0 & 110.9 & 116.4 & 106.2 & 106.7 \\
\hline TS+RT & 50.0 & 101.3 & 106.1 & 100.1 & 95.1 \\
\hline TS only & 49.7 & 101.3 & 104.7 & 99.5 & 95.6 \\
\hline RT only & 51.1 & 94.9 & 96.1 & 94.7 & 94.2 \\
\hline Bromocriptine & 43.7 & 106.4 & 114.6 & 109.3 & 109.9 \\
\hline Controls & 50.5 & 105.9 & 114.3 & 118.1 & 120.5 \\
\hline$F$ & n.s & n.s & $\mathrm{p}<0.002$ & $\mathrm{p}<0.002$ & $\mathrm{p}<0.001$ \\
\hline Analysis covaried for premorbid IQ & - & - & n.s & $\mathrm{p}<0.002$ & $\mathrm{p}<0.001$ \\
\hline
\end{tabular}

TF+RT = Transfrontal hypophysectomy and radiotherapy; TS+RT=trans-sphenoidal hypophysectomy and radiotherapy; TS only = trans-sphenoidal hypophysectomy only; RT only = Radiotherapy only; bromocriptine=bromocriptine drug treatment only in the absence of surgery or radiotherapy; controls=selected where possible from the family and friends of the patients

individual groups show that these effects were explained by the low pre-morbid and current IQ scores of the radiotherapy only group. This most probably results from a selection bias in these patients (in this small sample of radiotherapy only patients, a relatively high proportion had a low premorbid IQ). As a result, for many of our subsequent analyses, we covaried for the effect of premorbid IQ. None of the groups showed a decline in current IQ with respect to their estimated premorbid IQ (as determined by the NART-R IQ-WAIS-R IQ difference), and there were no significant differences between the groups in terms of current IQ after covarying for estimated premorbid IQ (NART-R). In brief, there was no evidence of a general decline in cognitive function in any of the groups.

By contrast, the anterograde memory quotients (table 2) indicated that there were highly significant differences between the groups for both general or "immediate" (verbal and nonverbal) memory and for delayed recall on the WMS-R, $(F(5,103)=4.07 \mathrm{p}<0.002$ and $F$ $(5,103)=7.11 \mathrm{p}<0.001$, respectively). All the groups, except the healthy controls, showed mean general and delayed memory quotients (GMQ, DMQ) which were several points below their mean intelligence quotients, and after covarying for estimated pre-morbid IQ

Table 3 Main outcome measures for treatment groups against healthy controls

\begin{tabular}{lll}
\hline & & $\begin{array}{l}\text { Covaried for premorbid } \\
I Q\end{array}$ \\
Treatment groups against healthy controls & $T$ & \\
\hline General memory (GMQ): & & 0.01 \\
$\quad$ Trans-frontal + radiotherapy $v$ controls & 0.001 & 0.001 \\
Trans-sphenoidal + radiotherapy $v$ controls & 0.001 & 0.001 \\
Trans-sphenoidal only $v$ controls & 0.001 & 0.01 \\
Radiotherapy only $v$ controls & $\mathrm{NS}$ & 0.05 \\
Bromocriptine only $v$ controls & & \\
Delayed memory (DMQ): & 0.01 & 0.001 \\
Transfrontal + radiotherapy $v$ controls & 0.001 & 0.001 \\
Trans-sphenoidal + radiotherapy $v$ controls & 0.001 & 0.001 \\
Trans-sphenoidal only $v$ controls & 0.001 & 0.005 \\
Radiotherapy only $v$ controls & $\mathrm{NS}$ & 0.025 \\
Bromocriptine only $v$ controls & & \\
IQ-MQ difference: & 0.001 & 0.005 \\
Transfrontal + radiotherapy $v$ controls & 0.05 & 0.05 \\
Trans-sphenoidal + radiotherapy $v$ controls & 0.05 & 0.05 \\
Trans-sphenoidal only $v$ controls & $\mathrm{NS}$ & $\mathrm{NS}$ \\
Radiotherapy only $v$ controls & 0.05 & 0.05 \\
Bromocriptine only $v$ controls & & \\
\hline
\end{tabular}

Table 4 Mean scores on main outcome measures for aetiology

\begin{tabular}{llllll}
\hline Aetiology & NART-R IQ & Current IQ & $\begin{array}{l}\text { General } \\
\text { memory }\end{array}$ & $\begin{array}{l}\text { Delayed } \\
\text { memory }\end{array}$ & $\begin{array}{l}\text { IQ-MQ } \\
\text { difference }\end{array}$ \\
\hline Acromegaly & 100 & 106 & 102 & 98 & 4.3 \\
Cushing's & 103 & 107 & 99 & 99 & 7.2 \\
Chromophobe & 106 & 111 & 103 & 101 & 7.4 \\
Prolactinoma & 103 & 109 & 104 & 102 & 5.1 \\
\hline
\end{tabular}

(NART-R), the differences remained significant across the groups for both the general and delayed memory quotients $(F(5,102)=4.16$ $\mathrm{p}<0.002$ and $F(5,102)=7.01 \mathrm{p}<0.001$ respectively).

The scores of the individual treatment groups were each compared with the healthy control group (using $t$ tests) and the results are shown in table 3. In terms of both general and delayed memory, all groups except the bromocriptine group showed a significantly poorer performance than the controls, and even the bromocriptine group showed significantly lower scores when the effect of estimated premorbid IQ was covaried out from these analyses. All treatment groups, except the radiotherapy only group, also showed a significant discrepancy between their current IQ and their general memory performance (IQ-GMQ difference), indicating a decline in anterograde memory relative to general intelligence.

The findings from the recognition memory test (RMT) were converted to age related percentile scores, because of the wide age ranges in our groups. The results corroborated those from the WMS-R in terms of anterograde memory in that significant differences were found across the groups for both face and word recognition memory $(F \quad(5,103)=4.83$, $\mathrm{p}<0.001$ and $F(5,103)=2.47, \mathrm{p}<0.05$ respectively). For face recognition memory, there was a somewhat anomalous result whereby the trans-sphenoidal only group scored higher than the controls but the other groups all performed worse than controls. For word recognition memory, all the individual patient groups performed worse than the controls, and this difference from controls was significant in the case of the two trans-sphenoidal groups, the radiotherapy only group, and the bromocriptine group. By contrast, there were no significant differences between the groups in terms of the further supplementary tests, including the attention/concentration quotient (measured as part of the WMS-R), retrograde memory (AMI), frontal/executive tasks, language, and speed of mental processing. There were also no significant differences across the psychiatric variables, although there was a trend for patients to show higher anxiety and depression scores than the healthy controls.

To examine more closely the effect of treatment on anterograde memory, we removed the healthy controls from the analysis and re-examined the findings for the WMS-R general and delayed memory quotients. There 
was no significant difference between the groups for general (or "immediate") memory, although there was a significant difference for delayed recall on a one way ANOVA ( $F$ $(4,85)=3.24, \mathrm{p}<0.02)$, such that the bromocriptine group were the least impaired. However, even this disappeared after covarying for estimated premorbid IQ (NART-R), indicating that, although our treatment groups all differed significantly from the healthy controls on anterograde memory tests (after covarying for NART-R), the patient groups did not differ significantly from one another in terms of memory performance. In brief, the more invasive treatments (trans-frontal surgery rather than trans-sphenoidal surgery; combined surgery and radiotherapy rather than either surgery or radiotherapy in isolation) did not necessarily result in a greater degree of cognitive impairment.

Consequently, it was important to examine whether the aetiology of the original tumour might be contributing to the memory deficits. Not surprisingly there were higher proportions of patients treated for acromegaly and Cushing's disease in the trans-sphenoidal hypophysectomy only group (38\% and $43 \%$ respectively), the radiotherapy only group $(20 \%$ and $50 \%$ ), and the combined surgery and radiotherapy groups $(39 \%$ and $39 \%)$, than in the bromocriptine group ( $6 \%$ and $0 \%)$. All of the patients were regrouped, therefore, in terms of their original tumour aetiology, rather than choice of treatment, and the neuropsychological test data were reanalysed. The groups were divided into patients with acromegaly (19 patients), Cushing's disease (22 patients), prolactinomas (23 patients), and chromophobe adenomas (26 patients). Table 4 shows that there were negligible differences in the mean test scores between the patients in terms of the different types of tumour. A two factor (treatment, aetiology) ANOVA was carried out with delayed memory (the most sensitive variable in the previous analyses) as the dependent variable, to examine the respective effects of treatment and aetiology. This disclosed a significant effect of treatment $(F(4,74)=4.06$, $\mathrm{p}<0.005)$ but not of aetiology $(F(3,74)=1.28$, NS) on delayed memory. The treatment by aetiology interaction effect was not significant $(F(8,74)=1.17, \mathrm{NS})$.

Finally, because radiotherapy might have a delayed effect on cognitive function, ${ }^{28} 29$ whereas the effect of surgery would be expected to be immediate, we compared patients whose radiotherapy or surgical treatment had occurred more or less than 5 years before the cognitive assessment. There were no significant differences between these patient groups on any of our major outcome measures.

\section{General discussion}

In an investigation of 90 patients treated for a pituitary adenoma during the previous 1 to 30 years, we obtained significant deficits in anterograde memory (WMS-R, RMT) across the treatment groups when their performance was compared with 19 healthy controls, consistent with the findings in the two case studies. These deficits varied in degree and on the WMS-R were smaller in the bromocriptine group than in the surgery and radiotherapy groups. Lower premorbid and current IQ scores were found in the radiotherapy only group, but this was considered to result from an unrelated selection bias in this particular group of patients. In fact, none of the patient groups showed a lower mean current IQ than mean estimated premorbid IQ, showing that the deterioration in memory function occurred in the context of intact intellectual performance. Consistent with this, the memory deficits on the WMS-R remained after covarying for premorbid IQ (although not on the RMT for words which, as a recognition rather than a recall measure, was less sensitive to change). Moreover, there were no significant deficits on any of the supplementary measures of cognitive function, such as attention and concentration, frontal and executive tasks, or language and speed of information processing. Neither were there any significant differences between the groups for psychiatric variables (anxiety, depression, personality traits), and covarying for these did not affect the overall outcome in memory measures. It is important to emphasise that the only measures to show significant differences were the two tests of anterograde memory (WMS-R, RMT).

On the other hand, when the individual treatment groups were compared, omitting the healthy controls and the bromocriptine group, no significant differences were seen between them on the memory measures, after the influence of pre-morbid IQ was statistically removed, indicating that the severity of cognitive impairment was not specifically related either to surgery or radiotherapy, or a combination of treatments. Similarly, there was no significant effect of whether treatment had occurred more or less than 5 years earlier. However, a comparison of the effect of treatment with the effect of underlying aetiology indicated a significant effect only for the first. Taken together, these results indicate that surgery and radiotherapy and (to a lesser extent) bromocriptine can all have a deleterious effect on anterograde memory.

Cognitive deficits have previously been reported in pituitary patients in a study by Grattan-Smith et $a l^{4}{ }^{4}$ but those authors were mainly interested in the effects of radiotherapy. Consistent with the present study, they found impairments on measures of anterograde memory, some tests of which overlapped with those in the present investigation, and the findings seemed to be unrelated to whether or not the patients had had radiotherapy. Their study used controls in hospital who had chronic medical conditions such as psoriasis and rheumatoid arthritis, yet their findings were broadly consistent with the present study using nonhospitalised controls. Peace et $a l^{30}$ in a study carried out at the same time as ours, reported impairments on executive tasks and one memory task, and they argued that radiotherapy did not contribute to the cognitive deficits that they obtained. However, their subgroups were smaller than ours (they had 36 
patients in total) and they did not include a "radiotherapy only" group who, in our study, clearly showed impairments. Ours is the largest and most detailed study of this topic to date, and the deficits in anterograde memory have emerged across several different measures, whereas other aspects of cognitive and psychological function seemed intact. Memory deficits have previously been reported with large midline tumours invading the diencephalon, ${ }^{5}$ and pituitary tumours with suprasellar extensions show similar effects. ${ }^{6}$ Neuropsychological studies of patients who have received radiotherapy for nasopharyngeal carcinoma have also found cognitive deficits when compared with newly diagnosed and untreated patients. ${ }^{12}$ However, Dons et $a l^{1}$ cited a patient who had a trans-sphenoidal hypophysectomy without postoperative radiotherapy, who manifested memory deficits which impaired her work but which showed improvement on treatment with desmopressin acetate (DDAVP) replacement therapy, suggesting a possible hormonal contribution to the disorder. Patient 1 in the present series showed a particularly severe memory deficit (a 49 point IQ-GMQ discrepancy), and we know that the structures affected by the $90 \%$ irradiation dose in her case included the mammillary bodies, the mammillothalamic tract, the anterior thalamus, and the fornix, all of which are known to be critical for memory formation. ${ }^{52}$ It is plausible that these structures could also have been affected in patients treated with surgery alone, thereby producing memory deficits of comparable severity to the radiotherapy only group, although the precise mechanism underlying this remains unclear.

In conclusion, memory deficits occur after treatment for pituitary adenomas, and pressure effects, surgery, radiotherapy, or endocrine changes may all contribute to these. The present study suggests that treatment factors are more important than aetiological variables in causing this memory deterioration and that radiotherapy alone and surgery alone produce deficits of comparable severity. To investigate this further, we are currently conducting a prospective study of newly diagnosed patients, examining cognitive function before and after treatment, in conjunction with whole brain MRI.

EG's work was supported by the Special Trustees of St Thomas's Hospital and the Medical Research Council. We thank Dr R Beaney and the staff of the radiotherapy departmen of St Thomas's Hospital, and Dr David Russell-Jones for kindly commenting on the paper, and all those who have provided background clinical information.

1 Lee PWH, Hung BKM, Woo EKW, et al. Effects of radiation therapy on neuropsychological functioning in patients with nasopharyngeal carcinoma. $f$ Neurol Neurosurg Psychiatry
1989;52:488-92.
2 Parkin AJ, Hunkin N. Memory loss after radiotherapy for nasal pharyngeal carcinoma: an unusual presentation of amnesia. Br f Clin Psychol 1991;30:349-57.

3 Kapur N, Ellison D, Parkin AJ, et al. Bilateral temporal lobe pathology with sparing of medial temporal lobe structures: lesion profile and pattern of memory disorder. Neuropsychologia 1993;32:123-51.

4 Gratton-Smith PJ, Morris JGL, Shores EA, et al. Neuropsychological abnormalities in patients with pituitary tumours. Acta Neurol Scand 1992;86:626-31.

5 Luria AR. The neuropsychology of memory. New York: John Wiley, 1976.

6 Lishman WA. Organic psychiatry: the psychological consequences of cerebral disorder. 2nd ed. Oxford: Blackwell, 1987.

7 Starkman MN, Schteingart DE. Neuropsychiatric manifestifications of patients with Cushing's syndrome. Arch Intern Med 1981;141:215-9.

8 Dorn LD, Burgess ES, Dubbert B, et al. Psychopathology in patients with endogenous Cushing's syndrome: atypical or patients with endogenous Cushing's syndrome: atypica

9 McGauley GA, Cuneo RC, Salomon F, et al. Psychological well-being before and after growth hormone treatment in adults with growth hormone deficiency. Horm Res 1990; (suppl 4):52-4.

10 Wechsler D. Wechsler adult intelligence scale — revised. London: Psychological Corporation, 1981.

11 Butters N, Salmon DP, Munro Cullum C, et al. Differentiation of amnesic and demented patients with the Wechsler memory scale-revised. Clinical Neuropsychologist 1988;2: 133-48.

12 Warrington EK. The recognition memory test. Windsor: NFER-Nelson, 1984

13 Shallice T, Evans ME. The involvement of the frontal lobes in cognitive estimates.Cortex 1978;14:294-303.

14 Nelson HE. A modified card-sorting test sensitive to frontal lobe deficits. Cortex 1976;12:313-24.

15 Benton AL. Differential behavioral effects of frontal lobe disease. Neuropsychologia 1968;6:53-60.

16 Newcombe F. Missile wounds of the brain. Oxford: Oxford University Press, 1969

17 McKenna P, Warrington EK. The graded naming test. Windsor: Nelson, 1983.

18 Baddeley A, Emslie H, Nimmo-Smith I. The speed and capacity of language-processing test. Bury St Edmunds: Thames Valley Test Company, 1982.

19 Gronwall D, Wrightson P. Delayed recovery of intellectual function after minor head injury. Lancet 1974;ii:605-9.

20 Christianson SA, Neppe V, Hoffman H. Amnesia and vegetative abnormalities after irradiation treatment: a case study. Acta Neurol Scand 1994;90:360-6.

21 Nelson HE, Willison JR. The national adult reading test. 2nd ed. Windsor: NFER-Nelson, 1991.

22 Kopelman MD, Wilson BA, Baddeley AD. The autobiographical memory inventory (manual). Bury St Edmonds: Thames Valley Test Company, 1990.

23 Beck AT, Ward CH, Mendelson M, et al. An inventory for measuring depression. Arch Gen Psychiatry 1961;4:61-571.

24 Speilberger CD, Gorsuch RC, Lushene RE. Manual for the state-trait anxiety inventory. Palo Alto, California: Consulting Psychologists Press, 1970.

25 Hunt SM, McKenna SP, McEwan J, et al. The Nottingham Health Profile: subjective health status and medical consultations. Soc Sci Med1981;15:221-9.

26 Roger D, Nesshoever J. Individual differences in delayed heart rate recovery following stress: the role of extraversion, neurotism, and emotional control. Personality Individual Differences 1988,4.721-6.

27 Eysenck HJ, Eysenck BG. Eysenck personality inventory manual. London: University of London Press, 1964.

28 Grattan-Smith PJ, Morris JGL, Langlands AO. Delayed radiation necrosis of the central nervous system in patients irradiated for pituitary tumours. I Neurol Neurosurg Psychiatry 1992;55:949-55.

29 Al-Mefty O, Kersh JE, Routh A, et al. The long term effects of radiation therapy for benign tumours in adults. $f$ Neurosurg 1990;73:502-12.

30 Peace KA, Orme SM, Thompson AR, et al. Cognitive dysfunction in patients treated for pituitary tumours. $\mathcal{F}$ Clin Exp Neuropsychol 1997;19:1-6.

31 Dons RF, House JF, Hood D. Assessment of desmopressinenhanced cognitive function in a neurosurgical patient. Military Medicine 1989;154:83-5.

32 Kopelman MD. The Korsakoff syndrome. Br f Psychiatry 1995;166:154-73.

33 Lezak MD. Neuropsychological Assessment. New York: Oxford University Press, 1983. 